\title{
Les hommes du Kébarien géométrique de Neve David, Mont Carmel (Israël)
}

\author{
The Geometric Kebaran human remains from Neve David, Mount Carmel, Israel
}

\author{
F. Bocquentin - I. Crevecoeur · B. Arensburg • D. Kaufman · A. Ronen
}

Résumé Deux sépultures d'adultes ont été découvertes sur le site Kébarien géométrique de Neve David (Israël) daté de 13400 et 12610 ans BP (non calibrées). Elles viennent étayer un corpus de sépultures épipaléolithiques du Levant encore très limité. La sépulture de Neve David 1, un individu de sexe masculin, livre l'une des plus anciennes attestations de matériel de broyage associé à l'inhumation. Le traitement des défunts annonce très clairement les pratiques funéraires natoufiennes qui leur succèdent. L'analyse morphométrique de l'adulte le mieux conservé et sa comparaison aux autres spécimens épipaléolithiques de la région enrichissent les données sur la variabilité anatomique déjà connue pour

F. Bocquentin $(\bowtie)$

UMR 7041, CNRS, 21, allée de l'Université,

F-92023 Nanterre cedex, France

e-mail : fanny.bocquentin@mae.u-paris10.fr

Centre de recherche français à Jérusalem,

UMIFRE 7 CNRS-MAEE, 3, rue Shimshon,

Baka, Jérusalem, Israël

I. Crevecoeur $(\square)$

Laboratoire d'Anthropologie des Populations du Passé,

UMR 5199 - PACEA / Université Bordeaux 1,

Bâtiment B8, Avenue des Facultés,

33405 Talence Cedex, France

e-mail : i.crevecoeur@pacea.u-bordeaux1.fr

B. Arensburg $(\square)$

Department of Anatomy and Anthropology,

Sackler School of Medicine, Tel Aviv University,

Ramat Aviv, Tel Aviv 69978, POB 39040, Israel

e-mail : arensbur@post.tau.ac.il

D. Kaufman $(\bowtie) \cdot$ A. Ronen $(\bowtie)$

Zinman Institut of Archaeology, university of Haifa,

Mount Carmel, 31905 Haifa, Israel

e-mail : dkaufman@research.haifa.ac.il,

aronen@research.haifa.ac.il

Cet article est dédié à la mémoire de notre ami Henri Laville, 1937-1995, découvreur du site de Neve David. cette période.

Mots clés Proche-Orient · Kébarien géométrique · Épipaléolithique · Sépultures · Site d'agrégation · Morphométrie $\cdot$ Matériel de broyage

Abstract The Geometric Kebaran site of Neve David, dated 13,400 and 12,610 BP (uncalibrated), revealed two burials of adults, adding to a still very limited corpus of Epipaleolithic inhumations in the Levant. Neve David 1, a male, is one of the oldest known burials accompanied by ground stone implements. The burials clearly reflect the funerary customs of the following Natufian culture. The morphometric analyses of the best-preserved adult in the context of other Epipalaeolithic specimens from the region add to the anatomical variability already documented for this period.

Keywords Near East - Geometric Kebaran · Epipalaeolithic - Graves - Aggregation camps · Morphometry $\cdot$ Ground stone implements

\section{Contexte chronoculturel : l'Épipaléolithique du Levant}

L'Épipaléolithique du Levant est la période comprise entre le Paléolithique supérieur et le Néolithique. Durant cette période, entre 21000 et $10500 \mathrm{Cal} \mathrm{BC}$ (toutes les datations calibrées sont données d'après Byrd [1]), trois entités archéologiques principales ont été reconnues : le Kébarien (21 000-16 000 Cal BC), le Kébarien géométrique (16 000$12900 \mathrm{Cal}$ BC) et le Natoufien (12 900-10 000 Cal BC), auquel succède le Néolithique ancien (10 000-8 650 Cal $\mathrm{BC})$. Il est généralement accepté que cette séquence représente un développement socioculturel continu qui a mené les derniers chasseurs-cueilleurs nomades à une économie basée sur l'agriculture au Néolithique [2-10]. 
L'Épipaléolithique du Levant a d'abord été défini à partir de ses industries. L'assemblage lithique de cette période est caractérisé par une production abondante de lamelles et d'outils microlithiques à retouche abrupte poursuivant ainsi une tendance déjà perceptible à la fin du Paléolithique supérieur. La production kébarienne inclut une variété de microlithes souvent courbes et à pointes étroites dans sa phase ancienne et, dans sa phase plus récente, de nombreuses lamelles à dos tronquées. Les triangles restent exceptionnels à cette période [5]. Les assemblages du Kébarien géométrique sont dominés par des produits de forme géométrique, principalement des trapèzes et des rectangles [2]. Au Natoufien, le segment de cercle devient le microlithe le plus fréquent. Il présente au début de la période une retouche bifaciale oblique (de type Helwan) remplacée, aux phases plus récentes, par une retouche abrupte. C'est au Natoufien qu'apparaissent les premières lames de faucilles. Le matériel de broyage se multiplie et se diversifie au Kébarien et tout au long de l'Épipaléolithique suggérant, d'une part, une diversification de l'usage de ce matériel en pierre et, d'autre part, une exploitation de plus en plus importante des ressources végétales $[11,12]$. Ces outils qui seront rapidement intégrés au mobilier funéraire [13] sont, au début de l'Épipaléolithique, essentiellement des mortiers profonds, des pilons ou des bols façonnés en calcaire ou en basalte. L'Épipaléolithique du Levant se caractérise aussi par le développement des échanges à longue distance, dont témoignent les matériaux exotiques. Le basalte provient de sources éloignées de plus de $100 \mathrm{~km}$ [14-17] et l'obsidienne d'Anatolie fait son apparition [18].

Les campements du Kébarien et du Kébarien géométrique diffèrent peu des campements du Paléolithique supérieur. Cependant, dès le Kébarien, on observe une dichotomie des types d'occupations avec, d'une part, des sites relativement étendus occupés dans la durée et, d'autre part, de petits campements éphémères [19]. Cela est interprété selon un modèle d'agrégation/dispersion, où sites logistiques et résidentiels participent à l'organisation du territoire [20]. Cette complémentarité est plus prononcée encore au Kébarien géométrique et devient une caractéristique fondamentale des occupations natoufiennes. En effet, au Natoufien une partie des groupes de chasseurs-cueilleurs se sédentarisent et édifient les premiers hameaux en pierre de la région. Cela s'accompagne d'une modification de l'exploitation des ressources environnementales [e.g.12,21]. Il est possible que les fondations d'un système social non égalitaire se mettent en place à la même période [e.g.19,22] .

\section{Une modification de l'espace funéraire à l'Épipaléolithique}

Les deux sépultures de Neve David sont attribuées au Kébarien géométrique, une période qui n'a livré pour le moment que très peu de restes humains. Parmi eux, rares sont ceux qui ont été trouvés en contexte funéraire. De la fin du Paléolithique supérieur à l'Épipaléolithique récent (ca 21000 à $12900 \mathrm{Cal} \mathrm{BC}$ ), seules sept autres sépultures ont été décrites jusqu'à présent dans la région à Nahal Ein Gev I [23], Ohalo II [24], Ayn Qasiyya [25], Ein Gev I [26], Wadi Mataha F-81 [27] et deux à Kharaneh IV [28]. Le site Kébarien géométrique d'Uyun Al-Hammam à lui seul vient rajouter à ce corpus 13 squelettes qui n'ont encore été que brièvement décrits $[29,30]$. Il semble donc que l'espace funéraire, s'il existait en tant qu'entité définie, se trouvait, à cette période, à distance des lieux de vie. Les rares sépultures découvertes en contexte d'habitat apparaissent d'emblée exceptionnelles. Les deux sépultures de Neve David, qui n'ont été que brièvement publiées lors de leur mise au jour [10,31], sont une source d'information incomparable sur une pratique funéraire encore marginale et une population épipaléolithique, dont on ne connaît que de rares représentants. Les groupes natoufiens, à partir de $12900 \mathrm{Cal}$ $\mathrm{BC}$, apportent une révolution dans le mode de traitement des morts qui semblent, de façon non systématique mais fréquente, associés à un habitat de plus en plus pérenne. Environ, 430 squelettes natoufiens ont été découverts jusqu'à présent. On constate que, désormais, le nombre de sépultures est en rapport (pas forcément proportionnel) avec la durée d'occupation et l'étendue du site (et bien sûr avec l'étendue des fouilles). Les sépultures témoignent d'une organisation nouvelle de l'espace où vivants et morts se côtoient. Le traitement des défunts est varié, mais répond à un certain nombre de règles communes à l'ensemble de l'entité natoufienne ou bien identifiées pour une période de temps ou une communauté donnée [32]. Si l'inhumation primaire et individuelle domine, le traitement collectif, multiple et les dépôts secondaires sont également présents. Des dépôts funéraires (parure, outils, restes sélectionnés d'animaux sauvages et de chiens domestiques) accompagnent parfois le défunt (pour une synthèse récente sur le sujet cf. Bocquentin [33]).

\section{Site de Neve David}

\section{Découverte et exploration du site}

Le site Kébarien géométrique de Neve David est situé au pied du versant ouest du Mont Carmel, sur la rive droite (nord) de l'embouchure du ouadi Siah (Nahal Siah), $60 \mathrm{~m}$ environ au-dessus du niveau de la mer et distant de $1 \mathrm{~km}$ du rivage actuel de la mer Méditerranée (Fig. 1). C'est Henri Laville (Université de Bordeaux 1) qui, en 1983, découvre le site lors de prospections des formations quaternaires sur le versant ouest du Mont Carmel. Auparavant, seule une occupation chalcolithique/âge du Bronze était connue à cet endroit 


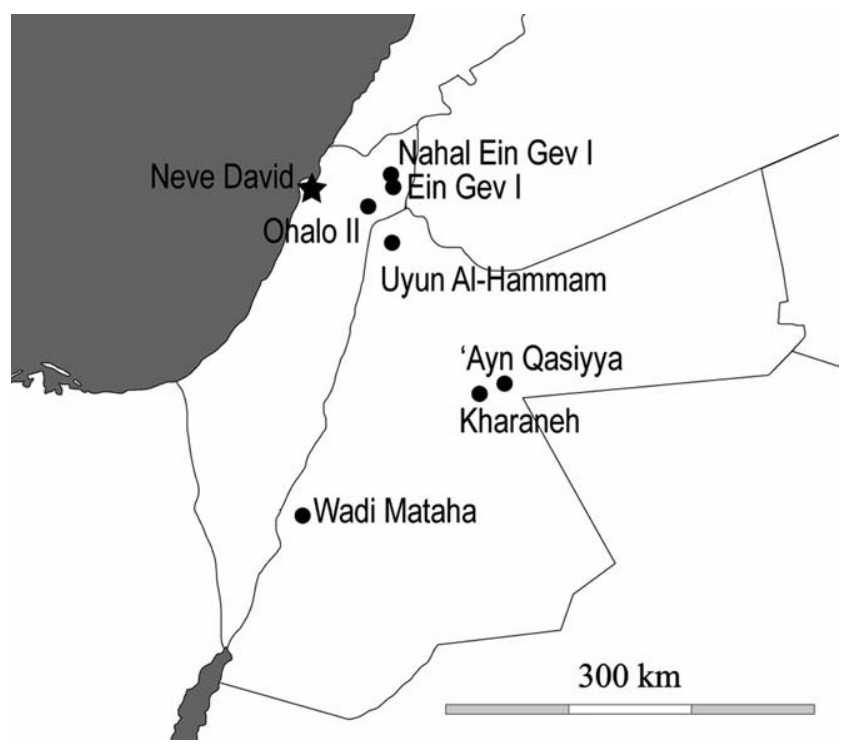

Fig. 1 Carte des sites Kébariens et Kébariens géométriques ayant livré des sépultures / Map of the Kebarian and Geometric Kebarian settlements with human burials

[34:24, site $\left.n^{\circ} 11\right]$, la couche épipaléolithique, plus profonde, n'ayant été exposée que lors de la construction d'une route peu avant la prospection menée par H. Laville (Fig. 2).

H. Laville décrit la stratigraphie suivante (Fig. 3) :

- ensemble 1 : dépôt à galets calcaires dans un sédiment argileux brun rouge ;

- ensemble 2 : sédiment brun rouge argileux pratiquement dépourvu d'éléments calcaires ;
- ensemble 3 : éléments calcaires plus petits dans un contexte de sédiment fin, possiblement la base de l'ensemble 2 ;

- ensemble 4 : conglomérat de roches calcaires à ciment carbonaté très dur ;

- ensemble 5 : dépôt calcaire blanc et meuble à fragments calcaires très hétérogènes ;

- ensemble 6 : sol d'altération brun/marron avec concrétions verticales blanchâtres.

Les ensembles 1 et 2 du côté nord du ouadi contiennent, d'après H. Laville, de nombreux outils de silex qui, selon lui, ne sont pas en place mais sont manifestement anciens : « il faudrait (...) essayer de savoir à quelle industrie ils appartiennent... »(Laville in letteris, 1982). Ces couches, qui viennent combler une dépression des concrétions sousjacentes, constituent les niveaux archéologiques chalcolithiques/âge du Bronze et Kébarien géométrique du site de Neve David. Les ensembles 4 à 6 sont des concrétions fortement cimentées visibles sur 3 à $4 \mathrm{~m}$ d'épaisseur. L'inversion du champ magnétique terrestre observée dans ces couches suggère une datation plus ancienne que $780 \mathrm{ka} \mathrm{BP}$ (Ron, communication personnelle).

En suivant cette découverte, une prospection de surface, ainsi qu'un rafraichissement des coupes ont permis de mieux cerner la période d'occupation du site et son importance. Une fouille a été menée de 1984 à 1990 sous la direction de D. Kaufman et A. Ronen, puis de D. Kaufman, et grâce au soutien de l'Institut d'Archéologie, de l'Université de Haïfa. Un sondage de $3 \times 4 \mathrm{~m}$ a d'abord permis de s'assurer que les couches archéologiques étaient bien en place, et la fouille a été étendue par la suite à $55 \mathrm{~m}^{2}$. Les décapages ont été menés

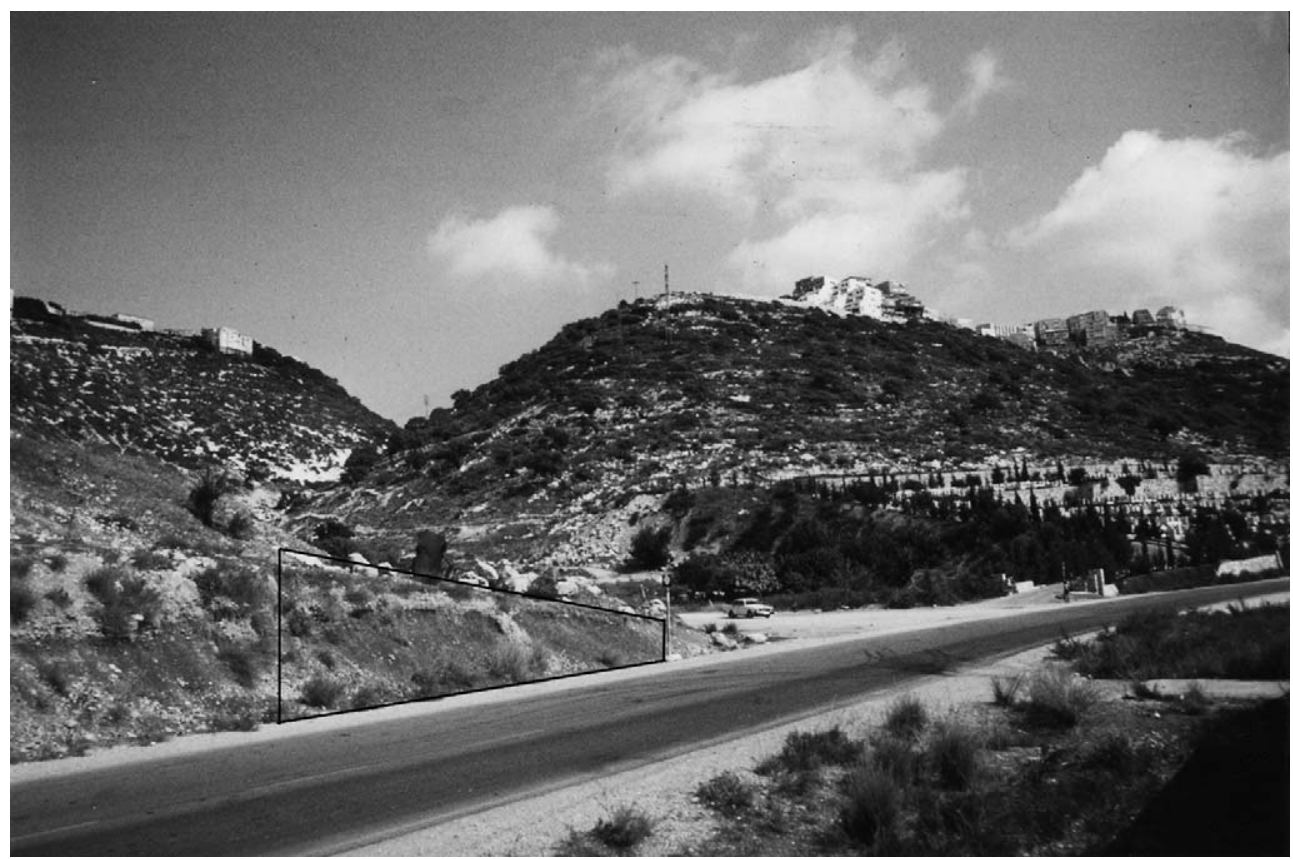

Fig. 2 Le site de Neve David photographié par Henri Laville en 1981 / The site of Neve David, photograph by Henri Laville in 1981 


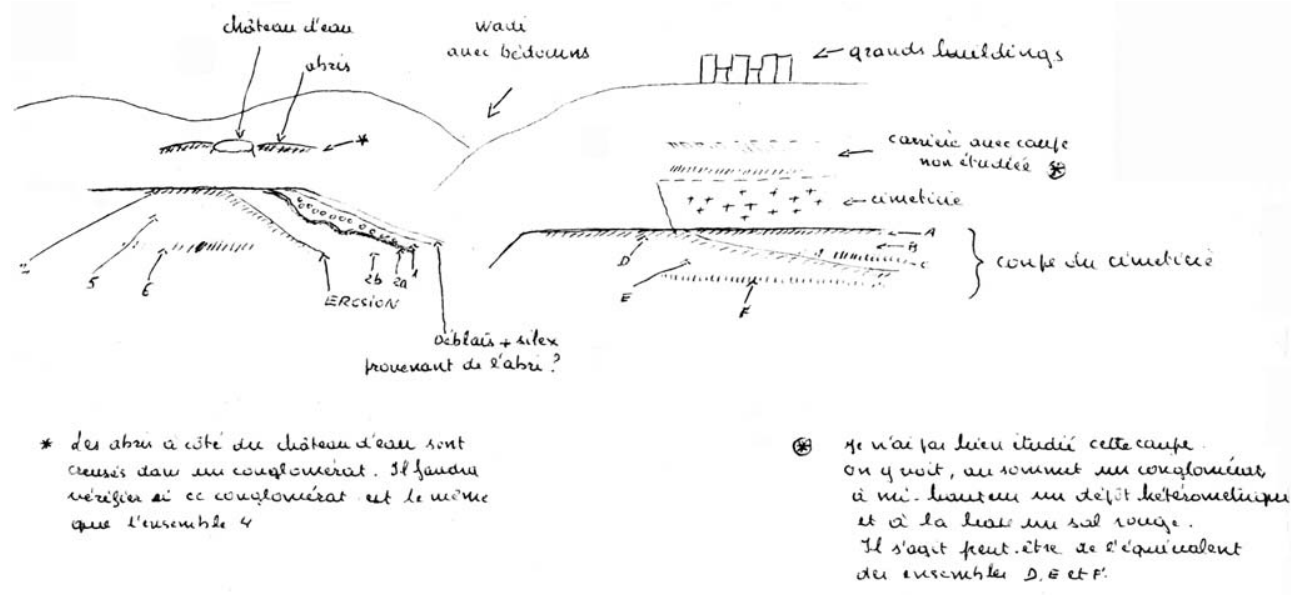

Fig. 3 Coupe du site de Neve David et annotations d'Henri Laville / Stratigraphy of Neve David with comments, by Henri Laville

par quart de mètre carré et par passes de $5 \mathrm{~cm}$. Tout le sédiment a été passé au tamis de $5 \mathrm{~mm}$.

\section{Description du site}

Neve David est localisé sur un écotone à l'interface de deux milieux environnementaux majeurs : les pentes du Mont Carmel à l'est et la plaine côtière à l'ouest qui, lors de l'occupation épipaléolithique, était 10 à $13 \mathrm{~km}$ plus large qu'à présent. L'approvisionnement en eau pouvait se faire par les nombreuses sources qui coulent le long du ouadi. Le silex se trouve en abondance dans le substrat calcaire du Mont Carmel, ainsi que dans le lit du ruisseau.

La surface totale du site est difficile à évaluer, mais il ne semble pas avoir été très étendu. Le long de la section faite par l'aménagement de la route, le matériel est visible sur $30 \mathrm{~m}$ dans le sens nord-sud, ce qui témoigne d'une importante destruction du niveau Kébarien géométrique. En considérant que l'extension du site dans le sens est-ouest était approximativement similaire, on peut estimer que l'occupation couvrait environ $1000 \mathrm{~m}^{2}$. L'importante densité de matériel, la présence de matériel de broyage lourd et de sépultures laissent penser que Neve David était un campement de regroupement d'une macrobande régionale évoluant selon un modèle de dispersion/agrégation saisonnier [8,9,19]. Durant son occupation, Neve David aurait donc servi de camp de base dans un système adapté à une mobilité logistique [Ibid., 20].

\section{Datations}

Deux échantillons d'os brûlés collectés à la proximité immédiate de la première sépulture (Neve David 1, ND1) ont permis d'obtenir deux datations de $12610 \pm 130 \mathrm{BP}$ (OxA-892 ; soit après calibration en tenant compte d'un écart type : 13 150-13 $450 \mathrm{Cal} \mathrm{BC}$ ) et $13400 \pm 180 \mathrm{BP}$ (OxA-859 ; soit après calibration en tenant compte d'un écart type : 14 820-14 090 Cal BC) [35]. Ces résultats posent problème dans la mesure où un écart de 800 ans sépare ces dates, et que même en tenant compte de deux écarts types, celles-ci ne se chevauchent pas. On peut suggérer qu'on a là le témoignage d'occupations successives, mais les deux échantillons proviennent du même endroit, et aucune division stratigraphique n'a été mise en évidence sur le terrain. Il est probable, toutefois, que si Neve David a bien été un site d'agrégation, il ait été occupé à plusieurs reprises, ce qui pourrait expliquer l'écart constaté entre les deux datations.

\section{Industrie lithique}

L'industrie lithique de Neve David [36] est caractéristique du Kébarien géométrique. L'assemblage (Tableau 1) est dominé par des lamelles et des nucléus unipolaires à lamelles. Les différents éléments de la chaîne opératoire incluant les préformes, lames, éclats, tablettes de ravivage, lames à crête sont présents, ce qui indique que la production a été menée sur le site. L'outillage est dominé par des microlithes géométriques $(40,6 \%)$ et non géométriques $(27,8 \%)$; les géométriques étant exclusivement représentés par des trapèzes (Tableau 2).

\section{Matériel de broyage}

Le matériel de broyage, diversifié, est façonné dans du basalte ou du calcaire. L'assemblage comprend des mortiers profonds, des bols, des meules, des molettes rondes et ovales. Deux de ces pièces étaient incluses dans une petite structure en pierre. Trois autres pièces, un mortier fracturé, un bol 


\begin{tabular}{|c|c|c|c|c|c|c|}
\hline & \multicolumn{2}{|l|}{ Débitage } & \multicolumn{2}{|l|}{ Outils } & \multicolumn{2}{|l|}{ Total } \\
\hline & Nombre & Pourcentage & Nombre & Pourcentage & Nombre & Pourcentage \\
\hline Nucléus & 264 & 3,2 & 1 & 0,1 & 265 & 3,0 \\
\hline Éclats & 2034 & 24,8 & 121 & 17,1 & 2155 & 24,2 \\
\hline Lames & 881 & 10,8 & 63 & 8,9 & 944 & 10,6 \\
\hline Lamelles & 3609 & 44,0 & 496 & 70,1 & 4105 & 46,1 \\
\hline Produits de mise en forme & 1187 & 14,5 & 22 & 3,1 & 1209 & 13,6 \\
\hline Tablettes de ravivage & 38 & 0,5 & 2 & 0,3 & 40 & 0,4 \\
\hline Produits d'entretien & 166 & 2,0 & 2 & 0,3 & 168 & 1,9 \\
\hline Autres & 17 & 0,2 & 1 & 0,1 & 19 & 0,2 \\
\hline Total & 8197 & 100 & 708 & 100 & 8904 & 100 \\
\hline
\end{tabular}

\begin{tabular}{|lcc|}
\hline $\begin{array}{l}\text { Tableau } 2 \text { Inventaire des pièces retouchées de Neve David } \\
\text { (d'après Shaul [36]) / Inventory of retouched tools from Neve } \\
\text { David (after Shaul [36]) }\end{array}$ \\
\hline Types & Nombre & Pourcentage \\
\hline Grattoirs & 76 & 10,7 \\
Burins & 20 & 2,8 \\
Perforateurs & 2 & 0,3 \\
Pièces tronquées & 7 & 1,0 \\
Encoches/denticulés & 40 & 5,6 \\
Outils multiples & 2 & 0,3 \\
Pièces retouchées & 68 & 9,6 \\
Microlithes non géométriques & 197 & 27,8 \\
Microlithes géométriques & 288 & 40,6 \\
Divers & 5 & 0,7 \\
\hline
\end{tabular}

fracturé et une plaquette à moudre étaient en association directe avec la sépulture ND1 (Fig. 4). Il s'agit d'un des plus anciens exemples d'objets de ce type découverts en contexte sépulcral au Levant. Ainsi, outre son importance économique, le matériel de broyage semble également être porteur d'une signification symbolique. Cela renforce la continuité suggérée entre le Kébarien géométrique et le Natoufien où la même association est constatée (cf. discussion).

\section{Faune}

L'assemblage faunique de Neve David (Tableau 3) est similaire aux autres sites épipaléolithiques prénatoufiens [37]. Les espèces les plus chassées sont la gazelle (Gazella gazella, $60 \%$ ) et le daim (Dama mesopotamica, $30 \%$ ). Les quelques autres grands mammifères présents sont l'aurochs (Bos primigenius), le cerf (Cervus elaphus) et le sanglier (Sus scrofa). Les petits mammifères sont représentés par quelques restes de

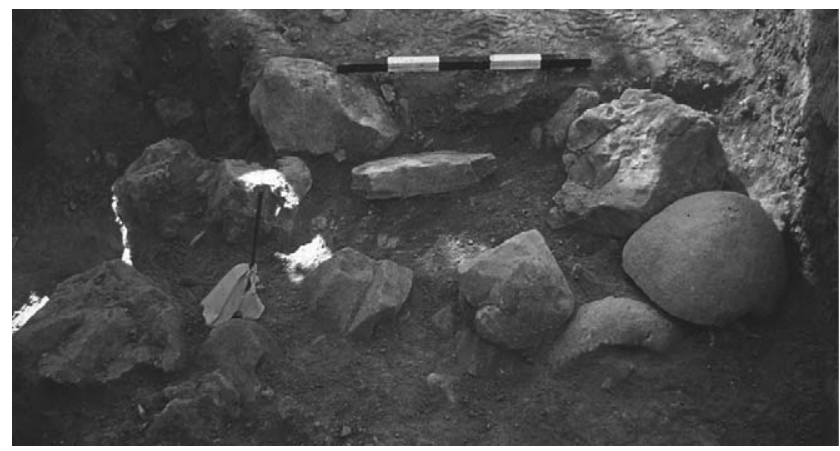

Fig. 4 Alignement de pierres au-dessus de la sépulture de Neve David 1. Photo prise du nord. En bas à droite : demi-mortier et demi-bol qui couvraient le haut du corps / Stone structure above the burial of Neve David 1. View from North. Below right: the halfmortar and half-bowl above the skull and against the shoulders

renards (Vulpes vulpes) et de lièvres (Lepus capensis). La tortue (Testudo graeca) était aussi exploitée.

\section{Les deux sépultures}

\section{Sépulture ND1}

La sépulture du premier adulte mis au jour (ND1) était recouverte de plusieurs gros blocs, assez grossièrement organisés en deux rangées et alignés sur 1,20 m d'ouest en est (Fig. 4). Parmi ces blocs, deux objets manufacturés en calcaire couvraient le haut du corps. Un demi-mortier fracturé longitudinalement, et percé par l'usure en son fond, avait été déposé directement sur la tête du défunt, " comme une sorte de casque en pierre, la partie concave en bas » $[31: 337]$. En outre, un demi-bol, fracturé de la même façon longitudinalement, avait été placé verticalement vraisemblablement contre sa colonne vertébrale. Celle-ci n'était pas conservée, mais son emplacement initial est déduit de la position du reste du 


\begin{tabular}{|c|c|c|}
\hline Espèces & NRD/NISP & NMI/MNI \\
\hline Gazella gazella & 1540 & 45 \\
\hline Bos primigenius & 13 & 1 \\
\hline Alcelaphus bucelaphus & 6 & 1 \\
\hline Dama mesopotamica & 780 & 21 \\
\hline Capreolus capreolus & 26 & 2 \\
\hline Cervus elaphus & 8 & 1 \\
\hline Sus scrofa & 9 & 2 \\
\hline Lepus capensis & 39 & 7 \\
\hline Vulpes vulpes & 41 & 3 \\
\hline Canis lepus & 10 & 1 \\
\hline Herpestes ischenum & 3 & 1 \\
\hline Martes foina & 1 & 1 \\
\hline Sciurus anomalous & 3 & 2 \\
\hline Hystrix indica & 1 & 1 \\
\hline Erinaceus europeus & 1 & 1 \\
\hline Testudo graeca & 12 & 4 \\
\hline Ophisaurus apodus & 2 & 1 \\
\hline Total & 2495 & 95 \\
\hline
\end{tabular}

squelette au moment de la fouille. Sous les blocs de pierre, le défunt, recroquevillé sur lui-même, n'occupait environ qu'une moitié de la structure, sa partie ouest. Le corps avait été inhumé sur le côté droit, les membres inférieurs ramenés à hauteur du thorax (Figs. 5,6). Au moins, un des avant-bras avait été placé également en flexion forcée, la main sans doute ramenée près du visage. La conservation partielle du squelette ne permet pas de préciser davantage la position initiale du cadavre. Un fragment de plaquette à broyer en basalte avait été placé entre ses deux cuisses et contre l'abdomen. Des coquilles de Mitra Cornicula ont été découvertes à proximité du cou et de la tête du défunt.

\section{Sépulture ND2}

Le second adulte (ND2) semble avoir été inhumé dans une simple fosse et aucun objet ne lui était associé [10]. Le corps, orienté sud-est/nord-ouest reposait sur le dos légèrement pivoté vers sa droite, côté vers lequel les membres inférieurs ont été ramenés en flexion, perpendiculairement à l'axe du corps (Figs. 7,8). La hanche gauche est restée maintenue en position verticale vraisemblablement contre la paroi de la fosse. Les os des membres inférieurs ont été sectionnés à hauteur des genoux et des chevilles par des fosses de l'âge du Bronze. Le membre supérieur droit est étendu le long du corps, de sorte que la main (dont il ne restait, à la fouille,

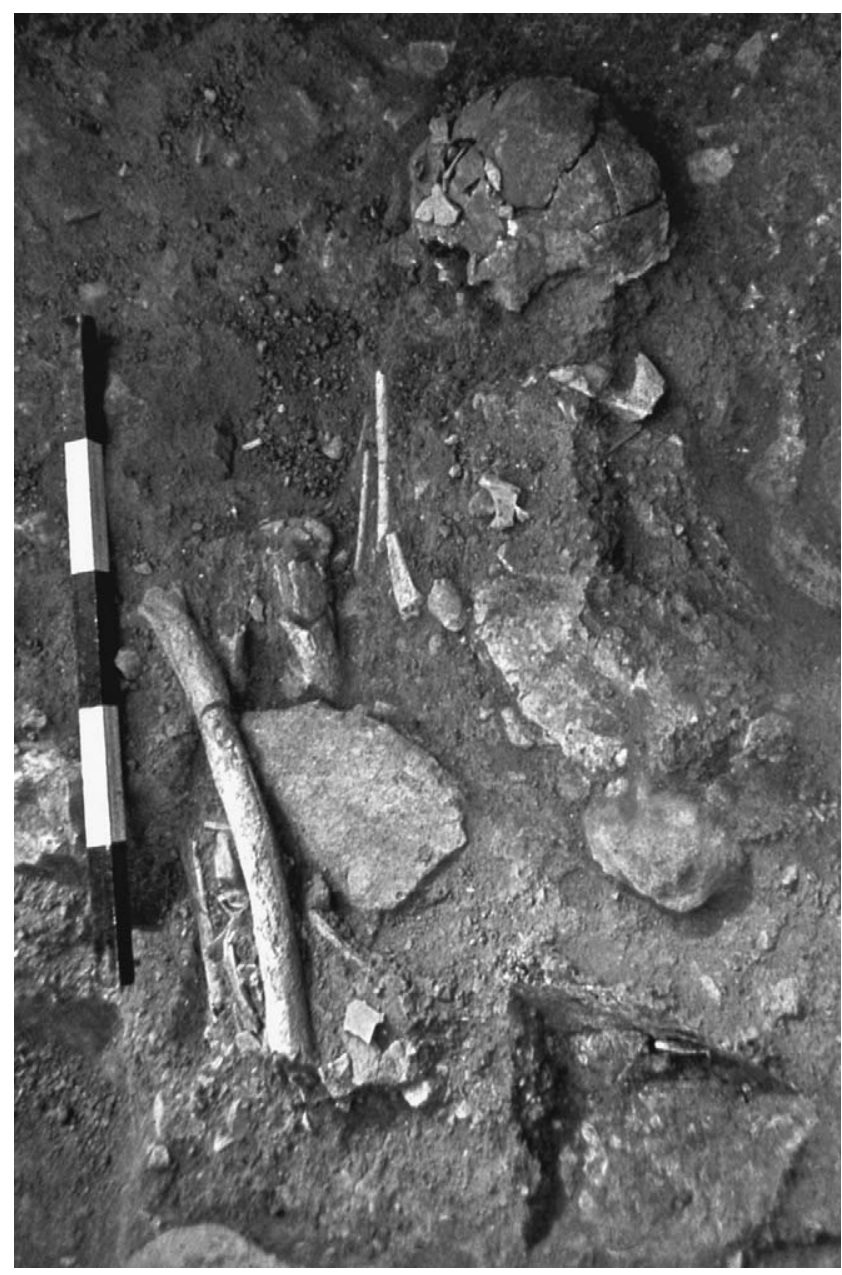

Fig. 5 Neve David 1 in situ après démontage des blocs de pierre / Neve David 1 in-situ after dismantling the stone structure

qu'un seul métacarpien) devait se trouver à hauteur des cuisses. Le membre supérieur gauche est fléchi à angle droit. La partie axiale supérieure du corps (thorax, cou et tête) n'est pas préservée. Le squelette est trop partiel pour que l'éventualité d'un prélèvement de crâne puisse être discutée. Un phénomène d'érosion est plus vraisemblablement à l'origine de sa disparition.

\section{Étude anthropologique}

\section{Conservation des squelettes}

Les deux squelettes de Neve David ne sont que très partiellement conservés. ND1 a souffert plus particulièrement des grosses pierres disposées directement sur son cadavre, et sous lesquelles, les os les plus fragiles ont disparu (os de la face, vertèbres, côtes et parties spongieuses des os longs) et les diaphyses des os les plus robustes ont été écrasées (en particulier les tibias). ND2 a souffert de 


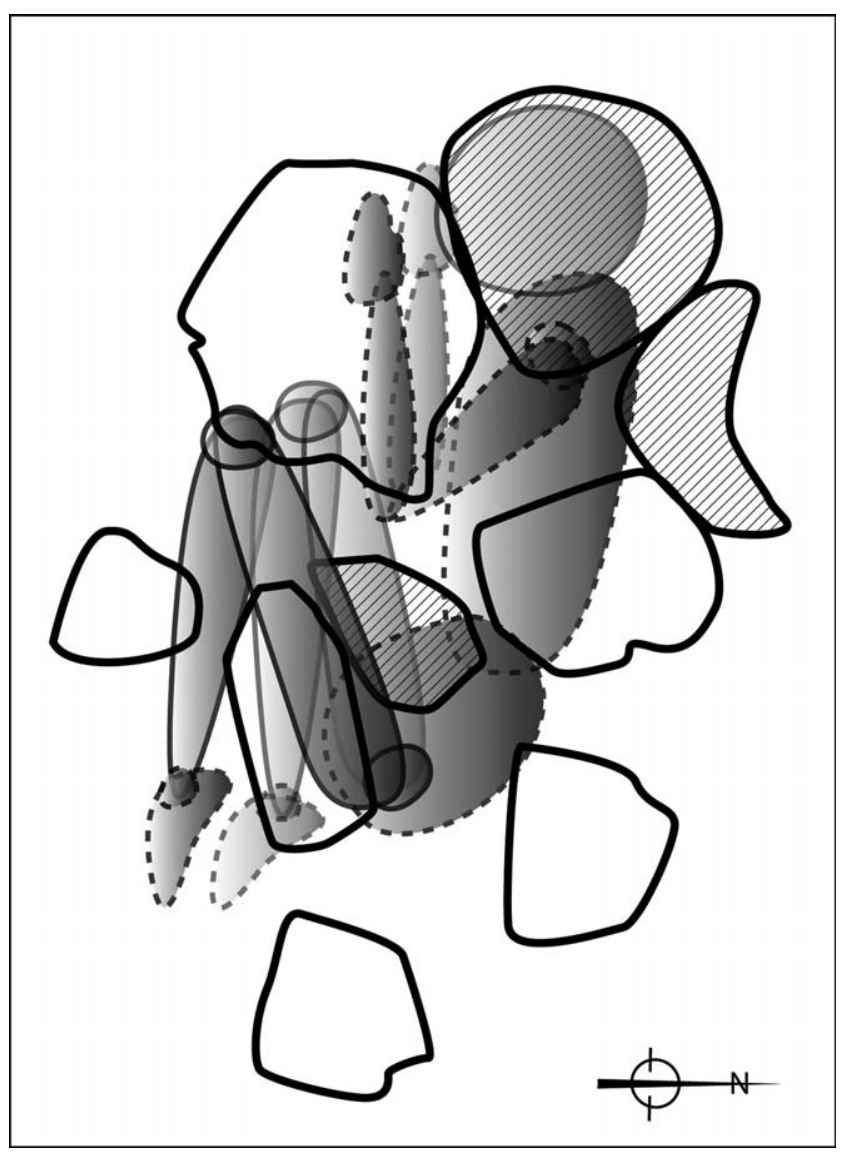

Fig. 6 Schématisation de la sépulture Neve David 1. Les pointillés différencient les parties manquantes du squelette. Le matériel de broyage associé étroitement au défunt apparaît hachuré / Schematic rendering of burial ND1. The dotted lines indicate the missing parts of the skeleton. Shading indicates the grinding tools directly in contact with the skeleton

perturbations tardives qui sont venues sectionner les extrémités des os longs et qui ont emporté le haut du corps. En outre, la découverte des squelettes est déjà ancienne et la collection n'est aujourd'hui que partielle ; ce qui reste des deux individus a été regroupé. ND2 n'est plus représenté que par quelques fragments de coxal (mature, sexe indéterminé). ND1 est mieux représenté ; les os de la collection qui lui appartiennent, avec certitude, sont schématisés sur la Figure 9. Le bloc craniofacial est représenté par une partie des pariétaux (sauf les régions sagittale et coronale), la partie postérieure des temporaux (jusqu'à la région des méats acoustiques externes) et l'écaille occipitale. Concernant la mandibule, seul un fragment gauche du corps (de la symphyse à la seconde molaire) est préservé. Les dents conservées sont $\mathrm{I} 2, \mathrm{P} 1, \mathrm{P} 2$ et $\mathrm{M} 1$. Le squelette infracrânien est aujourd'hui constitué d'un fémur gauche, des deux tibias, d'une fibula gauche, tous fragmentaires, ainsi que des deux talus, du calcanéus droit et du métatarsien $\mathrm{V}$ gauche.

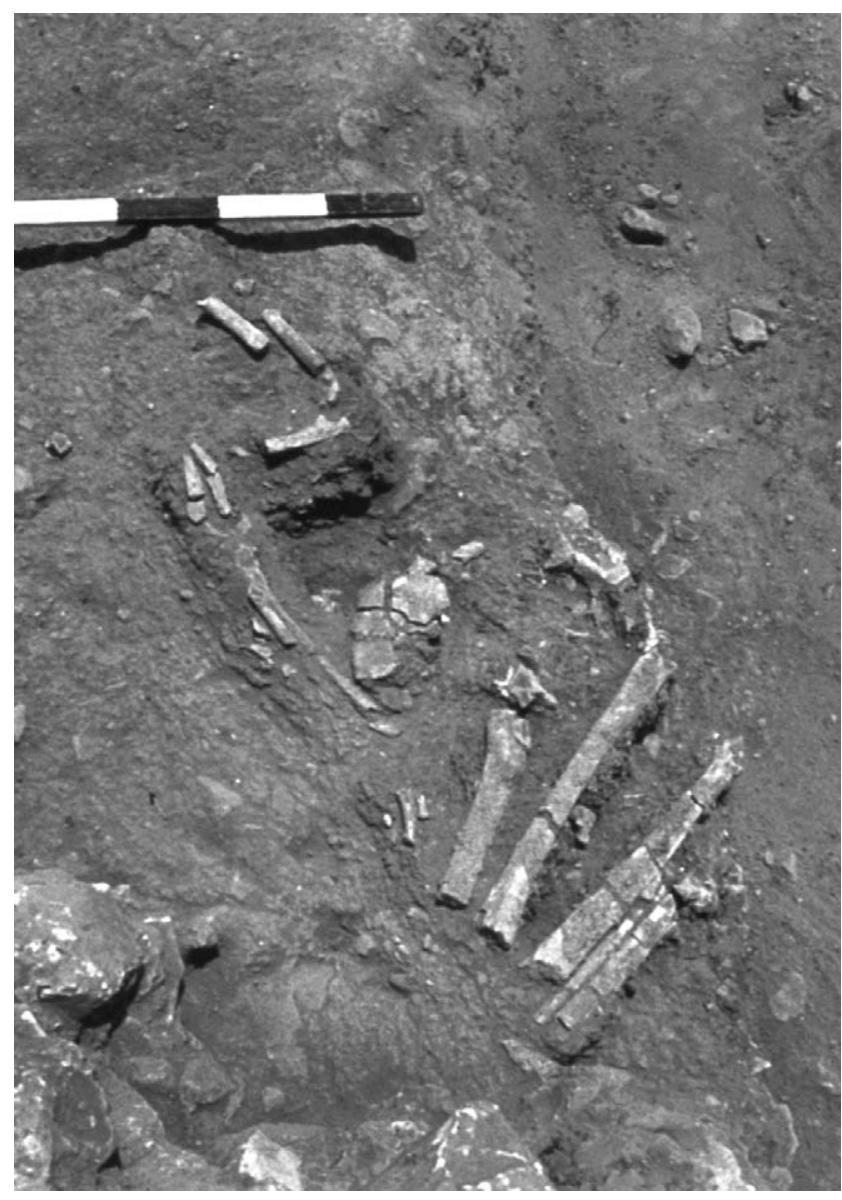

Fig. 7 Neve David 2 in situ / Neve David 2 in situ

Quelques échantillons ont été prélevés, afin d'examiner la préservation du collagène chez ND1 et ND2. Ils ont été envoyés au laboratoire Iom Beam Physics, ETH/PSI de Zurich ; aucun élément organique n'est préservé (Irka Hajdas, communication personnelle et aucune datation directe des restes ne peut être envisagée. Les ossements présents dans la collection sont tous concrétionnés, de la même façon que ce qui est observé dans les collections natoufiennes issues de sites de plein air. Un long travail de restauration pourrait permettre d'éliminer cette gangue calcaire afin, par exemple, d'observer d'éventuels caractères discrets ou les marqueurs musculaires d'activité. Nous nous sommes limités ici à une observation morphométrique des restes de Neve David.

\section{Analyse morphométrique et identité biologique de ND1}

Étant donné l'état actuel de la collection, seul ND1 se prête à une analyse morphométrique. Les dimensions crâniennes et infracrâniennes des restes ND1 apparaissent dans le Tableau 4 au côté des mesures individuelles publiées pour les autres spécimens du début de l'Épipaléolithique levantin, ainsi 


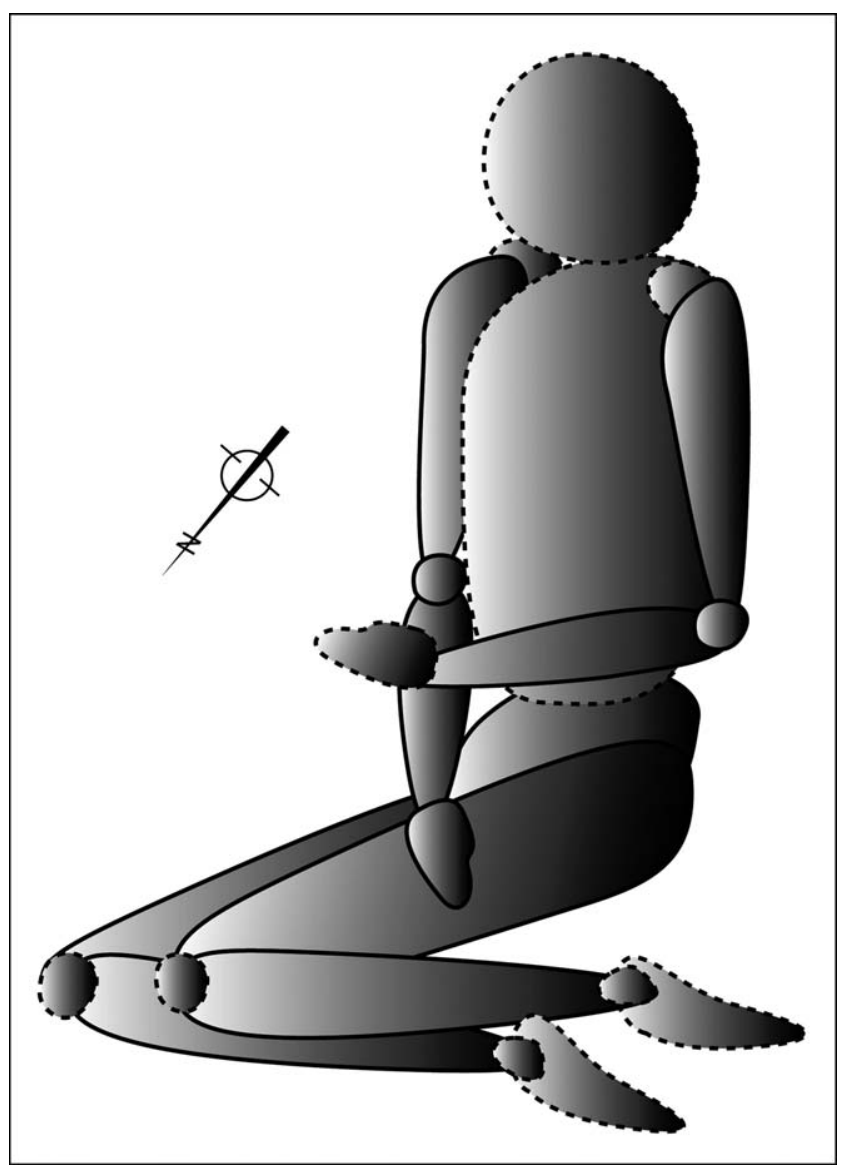

Fig. 8 Schématisation de la sépulture ND2. Les pointillés différencient les parties manquantes du squelette / Schematic rendering of burial ND2. The dotted lines indicate the missing parts of the skeleton

qu'au côté des données de synthèse natoufiennes. La technique des écarts réduits ajustés [38] a été utilisée pour apprécier la position de Neve David par rapport à la gamme de variation des Natoufiens, et ce, pour chaque mesure. D'autres populations de comparaison ont été utilisées occasionnellement, comme cela est mentionné plus en avant dans le texte.

\section{Bloc craniofacial et mandibule}

Le trait le plus immédiatement perceptible est la différence de robustesse entre ce qui reste du bloc craniofacial et le squelette infracrânien. Le bloc craniofacial et la mandibule sont remarquablement graciles (volume général, épaisseur de la voûte, reliefs absents, dents de petite dimension), mais les processus mastoïdes, en revanche, sont bien développés.

En ce qui concerne le neurocrâne, ND1 possède des largeurs de la voûte peu élevées qui se situent dans la partie inférieure de la variabilité natoufienne. Sa largeur bimastoïdes se situe même au-delà de la limite inférieure à $95 \%$ de la gamme de variation. Nous avons réalisé un diagramme

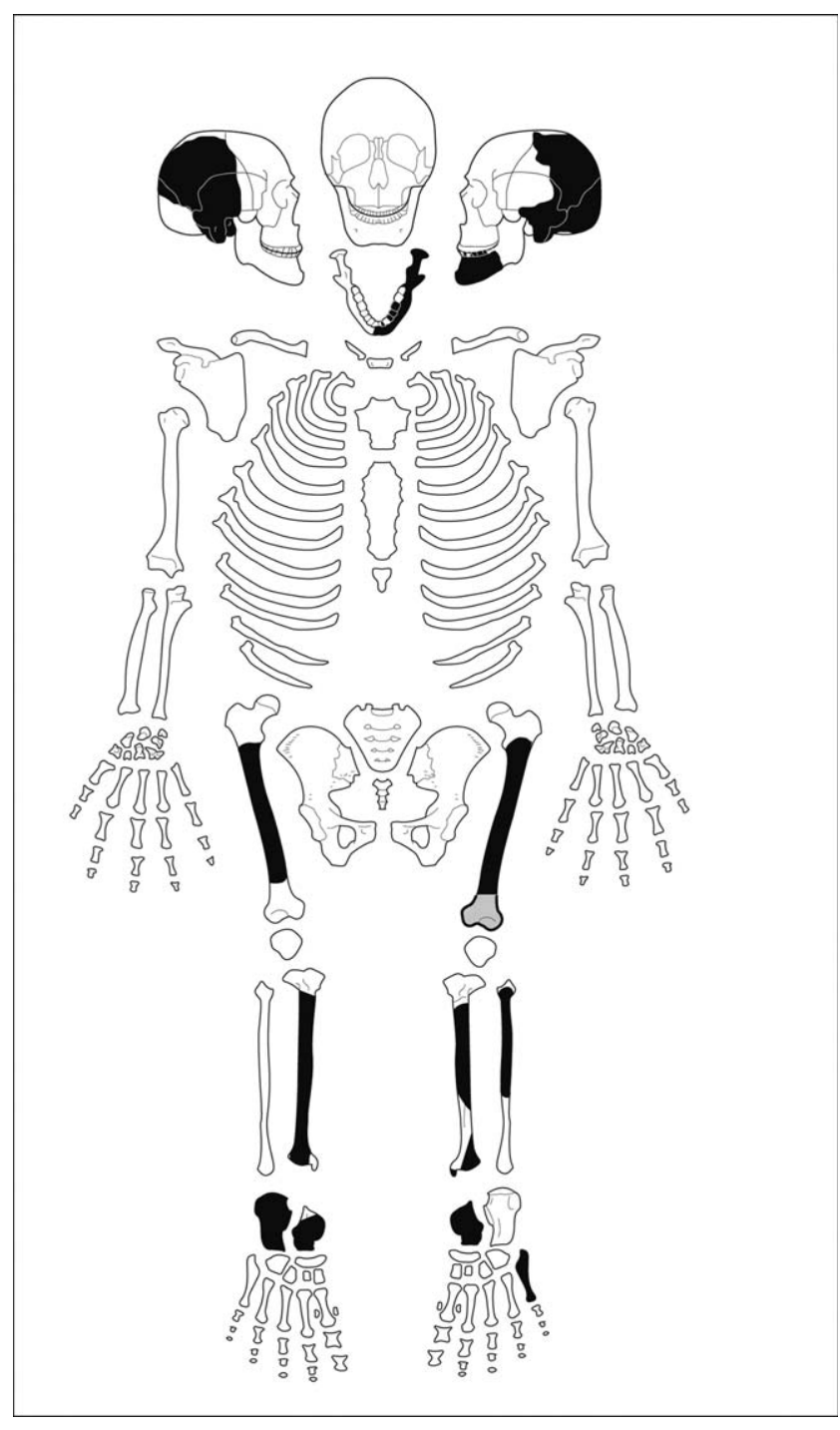

Fig. 9 Schéma de conservation du squelette Neve David 1. Seuls les os de la collection actuelle qui appartiennent avec certitude à cet individu ont été noircis / Outline of the preservation of skeleton Neve David 1. Bones from the current collection certainly belonging to that individual are shown in black

bivarié de la largeur maximale en fonction de la largeur biastérique en élargissant l'échantillon de comparaison, d'une part, aux séries épipaléolithiques du Maghreb et de Nubie que sont : Taforalt, Jebel Sahaba et Wadi Halfa et, d'autre part, aux hommes modernes actuels (Fig. 10). ND1 se place dans la partie inférieure du graphique en dessous des moyennes de tous les groupes épipaléolithiques, mais proche de celles de l'échantillon d'hommes modernes actuels.

La mandibule ND1 possède des hauteurs du corps qui se situent dans la gamme inférieure de la variabilité des Natoufiens. La hauteur à la symphyse est particulièrement faible par rapport à la moyenne natoufienne et aux autres individus Kébariens. De la même façon, les diamètres mésiodistaux, 


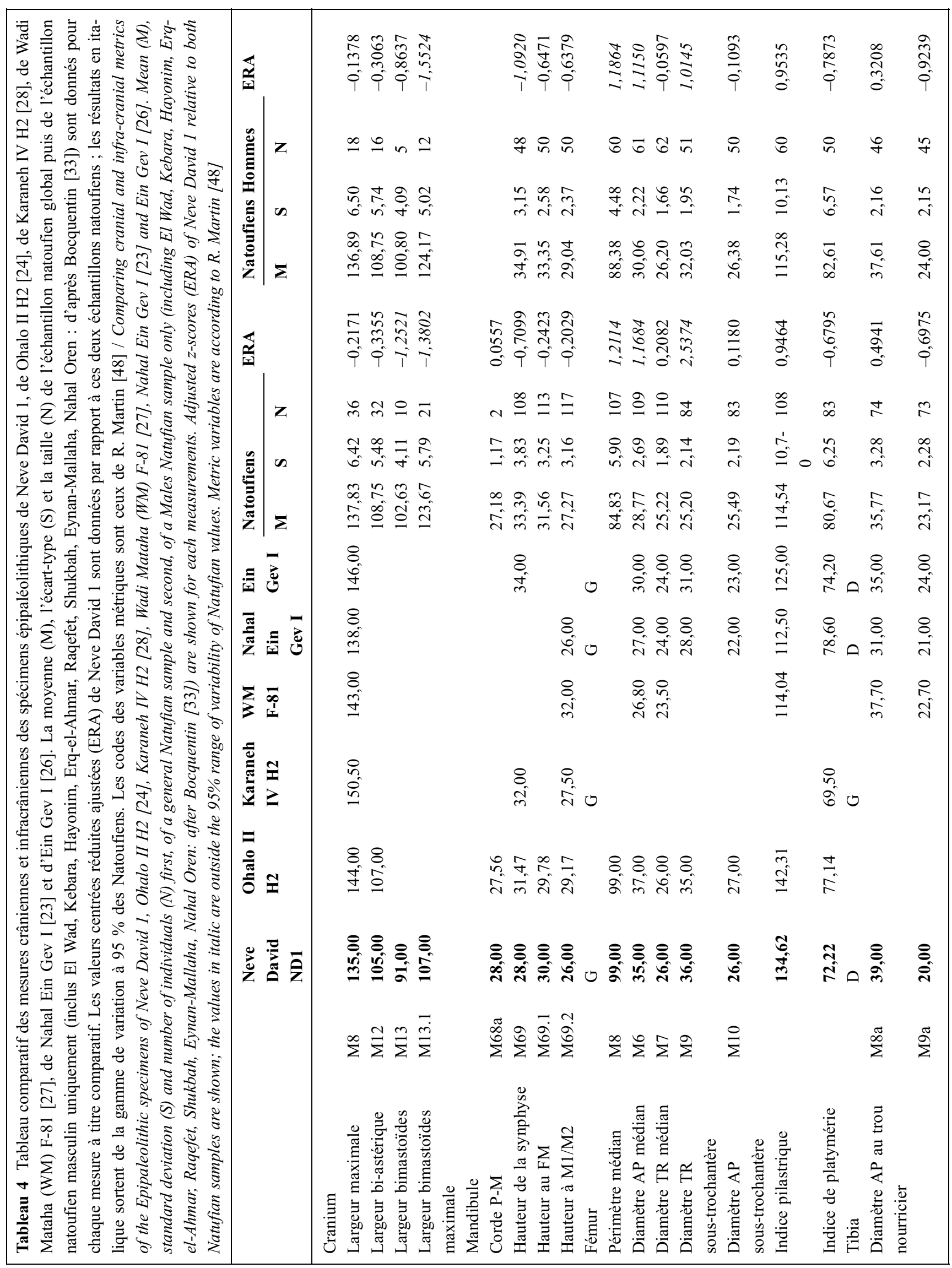




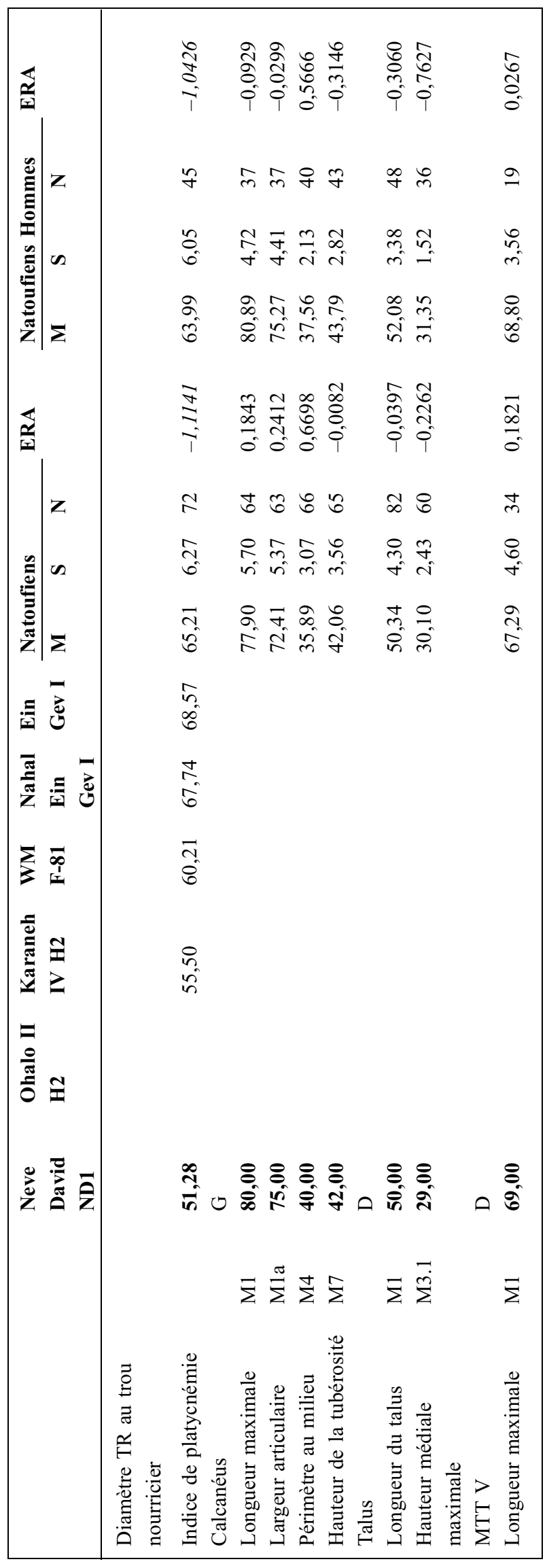

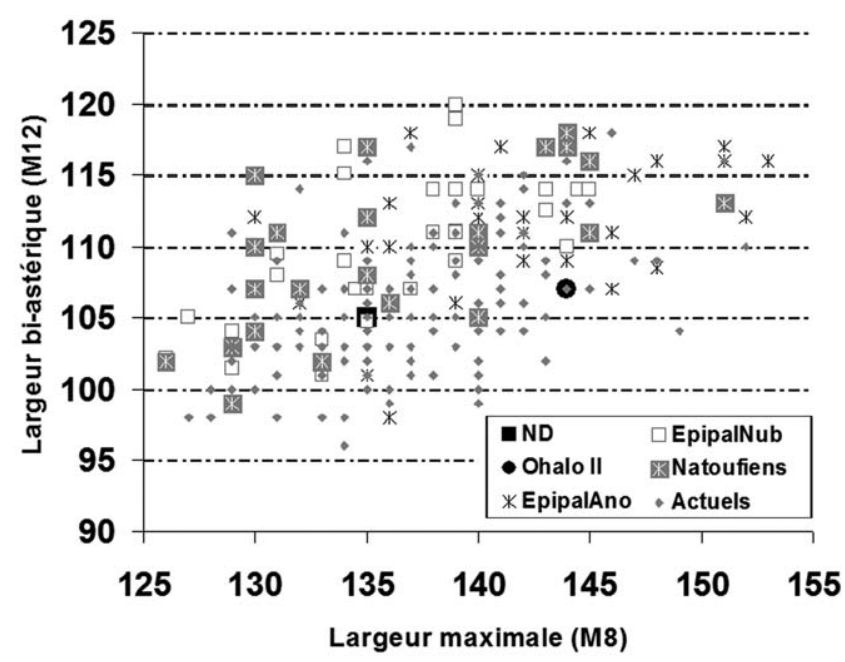

Fig. 10 Diagramme bivarié de la largeur maximale du crâne en fonction de la largeur bi-astérique / Bivariate plot of the maximal breadth (Eu-Eu) of the skull relative to the bi-asteric breadth (Ast-Ast) Légende : ND1 = Neve David 1 ; EpipalAno $=$ hommes modernes de l'Épipaléolithique du nord-ouest de l'Afrique (Taforalt) [46] ; EpipalNub = hommes modernes de l'Épipaléolithique nubien (Jebel Sahaba \& Wadi Halfa) [46] ; hommes actuels = groupe d'Afrique du Nord, Égyptiens [49]. Autres données : Ohalo II [24] ; Natoufiens [33] / Caption: ND1 = Neve David 1; EpipalAno $=$ Epipalaeolithic remains of North-West Africa (Taforalt) [46]; EpipalNub = Epipalaeolithic remains of Nubia (Jebel Sahaba \& Wadi Halfa) [46]; Actuels = Extant African sample from Egypt [49]. Other data: Ohalo II [24]; Natufians [33].

buccolinguaux et la hauteur des couronnes dentaires conservées sont de petites dimensions (Tableau 5). La Figure 11 représente les dimensions dentaires de Neve David, Wadi Mataha F-81, Ohalo II et Karaneh IV (H2) par rapport au groupe Natoufien. C'est plus particulièrement au niveau des diamètres buccolinguaux que les dents de ND1 apparaissent petites par rapport aux Natoufiens ; celui de la P1 est même hors de la gamme de variation natoufienne. Les diamètres de la M1 sont ceux qui se rapprochent le plus les différents spécimens prénatoufiens.

Les caractéristiques métriques du squelette crânien et des dents de ND1 témoignent de restes de faibles dimensions que ce soit par rapport à l'échantillon Natoufien, par rapport à d'autres séries épipaléolithiques d'Afrique du Nord ou aux spécimens pénécontemporains de la région.

\section{Restes infracrâniens}

Contrairement à la moyenne natoufienne et aux trois Kébariens auxquels il est comparé, le fémur (gauche) de ND1 possède un diamètre antéropostérieur très élevé à midiaphyse (Tableau 4). La valeur de l'indice pilastrique traduit 
Tableau 5 Dimensions des dents mandibulaires conservées sur Neve David 1 / Dental metrics of preserved mandibular teeth from Neve David 1

\begin{tabular}{|llllll|}
\hline ND1 Dents mandibulaires & & I2 & P1 & P2 & M1 \\
\hline Diamètre MD & M81 & 6,00 & 6,70 & 7,20 & 11,50 \\
Diamètre BL & M81,1 & 5,90 & 7,00 & 7,70 & 10,40 \\
Hauteur couronne & M81,2 & & 6,20 & 6,00 & \\
\hline
\end{tabular}

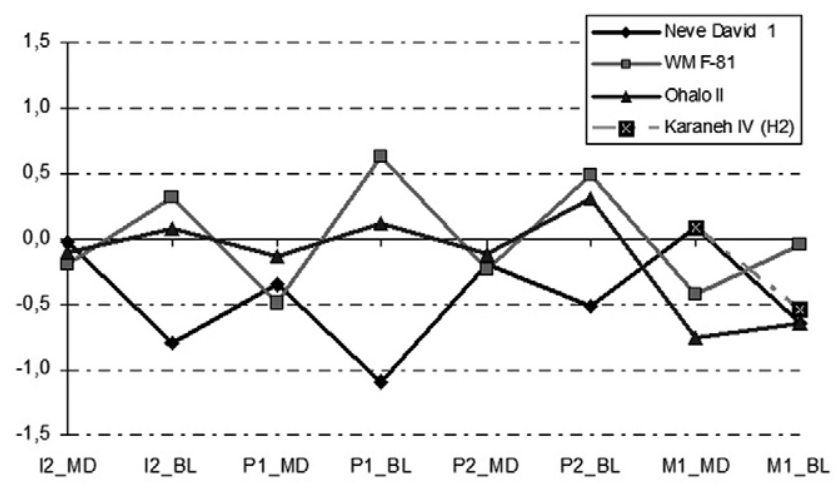

Fig. 11 Valeurs centrées réduites ajustées des diamètres mésiodistaux (MD) et buccolinguaux (BL) des dents mandibulaires conservées de Neve David 1 et des autres spécimens épipaléolithiques par rapport à l'échantillon natoufien [47]. L'intervalle entre +1 et -1 représente $95 \%$ de la variation des Natoufiens avec la ligne 0 comme moyenne. Mesures d'après : [27] (WM F-81) ; [24] (Ohalo II) ; [28] (Karaneh IV) / Adjusted z-scores of mesio-distal (MD) and buccolingual (BL) diameters of mandibular teeth from Neve David 1 and other Epipalaeolithic specimens relative to the Natufian sample [47]. Intervals between +1 and -1 show 95\% of the Natufian variability with the 0 line representing the mean value. Measurements from: [27] (WM F-81); [24] (Ohalo II); [28] (Karaneh IV)

par conséquent un pilastre fort, comme c'est le cas pour le fémur d'Ohalo II. De la même manière, le diamètre transverse sous-trochanter sort de la variabilité des Natoufiens et se rapproche de la valeur d'Ohalo II. La partie proximale de la diaphyse se caractérise par un contour asymétrique témoignant d'un aplatissement antéropostérieur de l'os. L'indice qui en résulte place ND1 au sein des fémurs hyperplatymériques.

Le tibia (droit) montre lui aussi un contour proximal très asymétrique. Le diamètre antéropostérieur au niveau de trou nourricier est très élevé. Son association avec un diamètre transverse faible place le tibia au sein des hyperplatycnémiques. La valeur de l'indice sort de la variabilité des Natoufiens. Seul le tibia de Karaneh IV H2 se rapproche du degré d'aplatissement transverse observé sur ND1. Cependant, dans le cas de Neve David, on ne peut exclure une déformation postmortem des tibias.
Le pied de ND1 est représenté par trois ossements complets, un calcanéus (droit), un talus (gauche) et un cinquième métatarsien (gauche) dont les dimensions s'inscrivent dans la gamme de variation de l'échantillon natoufien, du côté des valeurs élevées.

\section{Estimation du sexe}

Les os coxaux de ND1 n'étant pas conservés, une diagnose sexuelle primaire, c'est-à-dire qui tient compte du dimorphisme sexuel du pelvis commun à toutes les populations humaines, n'est pas possible. Une diagnose sexuelle à partir des autres os du squelette est possible à condition de disposer d'une population de référence jugée proche de Neve David, et dont le dimorphisme sexuel extracoxal est connu (diagnose probabiliste secondaire : cf. Murail et al. [39]). La population natoufienne nous a ainsi servi de référence [33]. Les analyses discriminantes qui permettent de séparer a posteriori avec une fiabilité de $100 \%$ (lorsque le seuil de décision est fixé à 0,90 ou plus de probabilité d'appartenir à l'un des sexes) les hommes et les femmes dont le sexe a été, au préalable, diagnostiqué par le coxal ont été appliquées à ND1. Les variables retenues sont celles observables sur ND1, et dont le pouvoir discriminant est le plus fort (Lambda de Wilk) chez les Natoufiens. Ainsi, à partir des mesures disponibles sur le fémur, le tibia et le calcanéus, les sept analyses discriminantes effectuées classent toutes ND1 parmi les hommes avec une probabilité supérieure à $97 \%$ (Tableau 6). Aucune analyse à partir des données crâniennes n'a été possible dans la mesure où cette région est très peu dimorphique dans la population natoufienne [33].

\section{Estimation de l'âge}

Les os conservés de ND1 suggèrent qu'on est en présence d'un squelette adulte (les épiphyses des os longs sont totalement soudées), mais l'absence des clavicules (dernier os du squelette à se souder) ne permet pas de préciser s'il s'agit d'un adulte jeune ou mature. La faible usure des dents (stade 2-3 sur l'échelle de Smith [40]), comparée à la vitesse d'usure des dents natoufiennes [33 : 581, annexe IIE], suggère toutefois que l'individu ne devait pas être très âgé. Aucune pathologie dentaire n'est présente. 
Tableau 6 Application de sept analyses discriminantes au squelette infracrânien de Neve David 1 pour une diagnose sexuelle secondaire [39]. Ces analyses discriminantes ont été calculées à partir des squelettes natoufiens dont le sexe a pu être déterminé grâce aux os coxaux [33]. (NFP : nombre de femmes natoufiennes participant au calcul ; NHP : nombre d'hommes natoufiens participant au calcul). LW : Lambda de Wilk (pouvoir discriminant de l'analyse) ; PBS : pourcentage des individus bien sexés avec une probabilité a posteriori d'appartenir au sexe réel supérieure au seuil de 0,90 (fiabilité de l'analyse). Dernière colonne : probabilité $a$ posteriori pour Neve David 1 d'être un homme sur la base de ses mesures extrapelviennes. Les codes des variables métriques sont ceux de Martin [48] / Seven selected discriminant analyses of infra cranial measurements employed on Neve David 1 for a probabilistic secondary sex determination [39]. These discriminant analyses were calculated on the basis of Natufian skeletons of known sex based on the morphometric characteristics of their pelvic bone (primary sex diagnosis) [33]. LW: Wilk's Lambda (discriminant power of the analysis); NFP: number of Natufian women participating; NHP: number of Natufian males participating; PBS: percentage of secondary correctly sex classified Natufian individuals (accuracy rate) with $p \geq 0.90$ versus primary sex diagnosis. Last column: posterior probability for Neve David 1 of being male on the basis of his extra-pelvic metrics. Measurement definitions from R. Martin [48]

\begin{tabular}{|c|c|c|c|c|c|c|c|c|c|c|c|}
\hline \multicolumn{11}{|c|}{ Analyses discriminantes secondaires (référence $=$ population natoufienne) } & \multirow{3}{*}{$\begin{array}{l}\text { Neve David } 1 \\
\text { Probabilité } \\
\text { sexe masculin }\end{array}$} \\
\hline $\mathbf{N}^{\circ}$ & Nombre & Nombre & \multirow[t]{2}{*}{ LW } & \multirow[t]{2}{*}{ NFP } & \multirow[t]{2}{*}{ NHP } & \multirow{2}{*}{$\begin{array}{l}\text { PBS } \\
\text { seuil } 0,90\end{array}$} & \multirow[t]{2}{*}{ Os } & \multicolumn{3}{|l|}{ Variables } & \\
\hline ADS & os & variables & & & & & & V1 & V2 & V3 & \\
\hline 37 & 2 & 2 & 0,374 & 10 & 15 & 100 & Fémur/tibia & F M8 & T M8a & & 0,999 \\
\hline 39 & 1 & 2 & 0,384 & 7 & 15 & 100 & Calcanéus & CAL M4 & CAL M7 & & 0,997 \\
\hline 51 & 1 & 2 & 0,408 & 10 & 14 & 100 & Tibia & $\mathrm{T}$ M8a & $\mathrm{T}$ M10b & & 0,969 \\
\hline 8 & 1 & 2 & 0,409 & 10 & 17 & 100 & Tibia & T M8a & T M9a & & 0,971 \\
\hline 24 & 1 & 2 & 0,41 & 7 & 14 & 100 & Calcanéus & CAL M1a & CAL M4 & & 0,993 \\
\hline 52 & 2 & 2 & 0,436 & 10 & 14 & 100 & Fémur/tibia & F M8 & $\mathrm{T}$ M10b & & 0,985 \\
\hline 1 & 1 & 3 & 0,588 & 12 & 22 & 100 & Fémur & F M6 & F M7 & F M8 & 0,993 \\
\hline
\end{tabular}

\section{Discussion}

Ce qui est préservé du squelette ND1 nous permet de dire que cet individu du Kébarien géométrique, de sexe masculin, présente une petite largeur crânienne comparée aux autres individus épipaléolithiques du Proche-Orient. Des dimensions crâniennes prénatoufiennes relativement réduites, par rapport aux Natoufiens, ont été observées par ailleurs [27], mais jamais en ce qui concerne la largeur crânienne. C'est plutôt en longueur et en hauteur que les prénatoufiens se distinguent de leurs successeurs [Ibid.]. Toutefois, les cinq squelettes de comparaison disponibles ne sauraient d'ores et déjà cerner la variabilité du début de l'Épipaléolithique. Concernant le squelette infracrânien, ND1 montre des affinités avec Ohalo II davantage qu'avec ses contemporains ou ses successeurs. Une activité physique intense comme une forte mobilité pourraient être à l'origine de ce parallèle anatomique [41].

Les positions d'inhumations observées à Neve David, sur le côté (ND1) ou de trois quarts (ND2) avec les membres inférieurs fléchis, sont proches de ce qui a été observé sur le site Kébarien d'Ein Gev I [26]. Toutefois, au Kébarien géométrique, la tendance dominante (d'après l'état actuel des connaissances qui s'appuie essentiellement sur le site d'Uyun Al-Hamman) est plutôt l'extension sur le dos [29,30]. La tête peut être relevée à la verticale comme dans le cas de Kharaneh IV [42] ou de la sépulture IV à Uyun AlHamman [30 : fig. 9]. L'extension sur le dos est une position qui perdure au début du Natoufien (uniquement en contexte d'inhumation en grotte) mais qui disparait par la suite. La position observée à Wadi Mataha (sur le ventre, jambes et avant-bras ramenés derrière le dos) apparait tout à fait exceptionnelle et interroge sur le statut du défunt (position du supplicié ?) [43]. En revanche, l'inhumation en position fléchie ou semi-fléchie, telle qu'on l'observe à Neve David, annonce une norme funéraire qui va perdurer pendant toute la durée du Natoufien [33].

Le dépôt de pierres directement sur le cadavre de ND1 a aussi été observé à Kharaneh IV [42]. C'est une pratique que l'on rencontre sporadiquement sur différents sites du Natoufien ancien et récent. De la même façon, la plaquette déposée entre les cuisses de ND1 a un écho dans les pratiques natoufiennes (sépulture H21 du Natoufien récent d'El Wad : [44 : PL. VI-4]). Mais le lien diachronique le plus évident est celui qui unit le défunt à du matériel de broyage cassé longitudinalement ou percé en son fond comme à Neve David ou à Wadi Mataha [27]. Il est clair que, dans le cas de Neve David, le mortier n'était plus adapté au broyage lors de son placement dans la sépulture, sa base ayant été percée vraisemblablement par une utilisation prolongée. On ne sait pas si l'objet a été accidentellement ou délibérément fracturé avant son dépôt dans la sépulture, mais il s'agit 
certainement d'un objet ayant perdu sa fonction première, et peut-être considéré comme « mort » au côté du défunt [13]. Au Natoufien, l'utilisation funéraire de mortiers, toujours en calcaire, percés ou fracturés, perdure notamment à El Wad [44] et à Nahal Oren [45], deux sites proches géographiquement de Neve David. Mais les mêmes gestes ont-ils encore la même signification?

Une filiation directe des individus de Neve David avec les Natoufiens est difficile à établir sur la base des données morphométriques, relativement atypiques de ND1. Les pratiques funéraires, en revanche, suggèrent l'existence d'un lien étroit entre les habitants de Neve David et leurs successeurs immédiats du Mont Carmel.

Remerciements Nous tenons à remercier Kürt Alt et Irka Hajdas qui ont eu l'amabilité d'analyser la conservation en collagène des ossements de Neve David. Nous remercions aussi Philippe Chambon pour ses pantins articulés fort utiles pour visualiser rapidement la position d'inhumation des défunts.

\section{Références}

1. Byrd BF (2005) Reassassing the emergence of village life in the Near East. J Archaeol Res 13:231-90

2. Bar-Yosef O (1981) The Epipaleolithic complexes in the southern Levant. In: Cauvin J, Sanlaville P (eds) Préhistoire du Levant. CNRS, Paris, pp 389-408

3. Bar-Yosef O (1990) The last glacial maximum in the Mediterranean Levant. In: Gamble C, Soffer O (eds) The World at 18,000 BP. Unwin Hyman, London, pp 58-77

4. Bar-Yosef O, Belfer-Cohen A (1989) The origins of sedentism and farming communities. J World Prehist 3:447-98

5. Bar-Yosef O, Vogel JC (1987) Relative and absolute chronology of the Epipaleolithic in the southern Levant. In: Aurenche O, Evin J, Hours F (eds) Chronologies in the Near East. BAR International Series 379, Oxford, pp 219-46

6. Henry DO (1983) Adaptive evolution within the Epipaleolithic of the Near East. Adv World Archaeol 2:99-160

7. Henry DO (1989) From foraging to agriculture: the Levant at the end of the ice Age. University of Pennsylvania Press, Philadelphia, $277 \mathrm{p}$

8. Kaufman D (1986) A reconsideration of adaptive changes in the Levantine Epipaleolithic. In: Strauss LG (ed) The end of the Palaeolithic in the Old World. BAR International Series 284, Oxford, pp 117-28

9. Kaufman D (1987) Excavations at the Geometric Kebaran site of Neve David, Israel: a preliminary report. Quartär 37-38:189-99

10. Kaufman D (1989) Observations on the Geometric Kebaran: a view from Neve David. In: Bar-Yosef O, Vandermeersch B (eds) Investigations in South Levantine Prehistory. Préhistoire du Sud Levant. BAR International Series 497, Oxford, pp 275-85

11. Wright KI (1994) Ground-stone tools and hunter-gatherer subsistence in Southwest Asia: implications for the transition to farming. Am Antiq 59:238-63

12. Dubreuil L (2004) Long-term trends in Natufian Subsistence: a use-wear analysis of ground stone tools. J Archaeol Sci 31:1613-19

13. Ronen A (2003) Grinding Tools as Grave Goods. In: Derwich E (ed) Préhistoire des pratiques mortuaires, Eraul 102, Liège, pp 63-8
14. Weinstein-Evron M, Lang B, Ilani S, et al (1995) K/Ar dating as a means of sourcing Levantine Epipaleolithic basalt implements. Archaeometry 37:37-40

15. Weinstein-Evron M, Lang B, Ilani S (1999) Natufian trade/ exchange in basalt implements: evidence from northern Israel. Archaeometry 41:267-73

16. Weinstein-Evron M, Kafuman D, Bird-David N (2001) Rolling stones: basalt implements as evidence for trade/exchange in the Levantine Epipaleolithic. Mitekufat Haeven. J Isr Prehist Soc 31:25-42

17. Ronen A (2010) The symbolic use of Basalt in the Levantine Epipalaeolithic and the emergence of socioeconomic leadership. In: Benz M (ed) The Principle of Sharing, segregation and construction of social identities at the transition from foraging to farming. Proceedings of the SIGN-Conference, Freiburg 2009

18. Valla FR, Khalaily H, Valladas H, et al (2007) Les fouilles de Ain Mallaha (Eynan) de 2003-2005 : quatrième rapport préliminaire. Mitekufat Haeven. J Isr Prehist Soc 37:135-380

19. Kaufman D (1992) Hunter-gatherers of the Levantine Epipaleolithic: the socioecological origins of sedentism. J Mediterr Archaeol 5:165-201

20. Binford LR (1980) Willow Smoke and Dogs' Tails: huntergatherer Settlement Systems and Archaeological Site Formation. Am Antiq 45(1):4-20

21. Munro ND (2004) Zooarchaeological measures of hunting pressure and occupation intensity in the Natufian. Implications for agriculture origins. Curr Anthropol 45:S5-S33

22. Bar-Yosef O, Belfer-Cohen A (2000) Early sedentism in the Near-East; a bumpy ride to village life. In: Kuijt I (ed) Life in Neolithic farming communities social organization, identity, and differentiation. Kluwer Academic/Plenum, New York, pp 19-62

23. Arensburg B (1977) New Upper Paleolithic human remains from Israel. Eretz Israel 13:208-15

24. Hershkovitz I, Speirs MS, Frayer D, et al (1995) Ohalo-II H2 a 19,000-year-old skeleton from a water-logged site at the Sea of Galilee, Israel. Am J Phys Anthropol 96:215-34

25. Richter T, Stock JT, Maher L, et al (2010) An Early Epipalaeolithic sitting burial from the Azraq Oasis, Jordan. Antiquity $84: 321-34$

26. Arensburg B, Bar-Yosef O (1973) Human remains from Ein Gev I, Jordan Valley, Israel. Paléorient 1:201-6

27. Stock JT, Pfeiffer S, Chazan M, et al (2005) F-81 skeleton from Wadi Mataha, Jordan, and its bearing on human variability in the Epipaleolithic of the Levant. Am J Phys Anthropol 128(2):453-65

28. Rolston SL (1982) Two prehistoric burials from Qasr Kharaneh. Annu Dep Antiq 26:221-9

29. Maher L (2007) Microliths and mortuary practices: new perspectives on the Epipaleolithic in northern and eastern Jordan. In: Levy TE, Daviau M, Younker MW, Sharer M (eds) Crossing Jordan: North American contributions to the Archaeology of Jordan. Equinox, London, pp 195-202

30. Maher L (n.d.) Recent excavations at a Geometric Kebaran Site in Wadi Ziqlab, Northern Jordan. Unpublished excavation report, http://www.bioanth.cam.ac.uk/lches_lm.html

31. Kaufman D, Ronen A (1987) La sépulture kébarienne géométrique de Neve David, Haïfa, Israël. L'Anthropologie 91:335-42

32. Bocquentin F, Chambon $\mathrm{P}$, Le Goff I, et al (on line) De la récurrence à la norme: interpréter les pratiques funéraires en préhistoire. Bull Mém Soc Anthropol Paris 22(1-2)

33. Bocquentin F (2003) Pratiques funéraires, paramètres biologiques et identités culturelles au Natoufien : une analyse archéoanthropologique. Thèse de doctorat en anthropologie biologique. Université Bordeaux-I, Talence, 629 p. http://ori-oai.u-bordeaux1.fr/ ori-oai-search/notice.html?id=u-bordeaux1-ori$163 \&$ format $=$ dc id 
34. Olami Y (1984) Prehistoric Carmel. Israel Exploration Society, Jerusalem, $216 \mathrm{p}$

35. Kaufman D (1988) New radiocarbon dates for the Geometric Kebaran. Paléorient 14:107-9

36. Shaul N (1999) The Geometric Kebaran Site in Neve David. M.A. Thesis, university of Haifa (Hebrew with English abstract)

37. Bar-Oz G, Dayan T, Kaufman D (1999) The Epipalaeolithic faunal sequence in Israel: a view from Neve David. J Archaeol Sci 26:67-82

38. Maureille B, Rougier H, Houët F, et al (2001) Les dents inférieures du Néandertalien Regourdou 1 (site de Regourdou, commune de Montignac, Dordogne) : analyses métriques et comparatives. Paléo 13:183-200

39. Murail P, Bruzek J, Braga J (1999) A new approach to sexual diagnosis in past populations. Practical adjustments from Van Vark's procedure. Int J Osteoarchaeol 9:39-53

40. Smith BH (1984) Patterns of molar wear in hunter-gatherers and agriculturalists. Am J Phys Anthropol 63:39-56

41. Stock JT (2006) Hunter-gatherer postcranial robusticity relative to patterns of mobility, climatic adaptation, and selection of tissue economy. Am J Phys Anthropol 131(2):194-204

42. Muheisen M (1988) The Epipaleolithic phases of Kharaneh IV. In: Garrard AN, Gebel HG (eds) The Prehistory of Jordan: the state of research in 1986. BAR International Series, 396, Oxford, pp 353-67

43. Guilaine J, Zammit J (2001) Le sentier de la guerre. Visage de la violence préhistorique. Éditions du Seuil, Paris, $377 \mathrm{p}$

44. Garrod DAE, Bate DMA (1937) The Stone Age of Mount Carmel: Volume 1. Clarendon Press, Oxford, $240 \mathrm{p}$

45. Stekelis M, Yizraeli T (1963) Excavations at Nahal Oren: Preliminary report. Isr Explor J 13:1-12

46. Crevecoeur I (2008) Étude anthropologique du squelette du Paléolithique supérieur de Nazlet Khater 2 (Égypte). Apport à la compréhension de la variabilité passée des hommes modernes. Egyptian Prehistory Monographs 8, Leuven University Press.

47. Smith P (1979) Regional diversity in epipaleolithic populations. Int J Skeletal Res (Ossa) 6:243-50

48. Bräuer G (1988) Osteometrie. In: Knussman R (ed) Anthropologie. Handbuch der vergleichenden Biologie des Menschen, 4. Auflage des Lehrbuchs des Anthropologie begründet von R Martin. Band I. Wesen und Methoden der Anthropologie, Gustav Fisher Verlag, Stuttgart, pp 160-231

49. Howells WW (1989) Skull shapes and the map: craniometric analysis in the dispersion of modern homo. Papers of the Peabody Museum, Harvard University Press, Cambridge, $187 \mathrm{p}$ 